\title{
Une esthétique agonale de la Grande Guerre
}

Eine agonistische Ästhetik des Ersten Weltkriegs

An agonistic aesthetic of the Great War

Jochen Mecke

\section{OpenEdition}

Journals

Édition électronique

URL : http://journals.openedition.org/ceg/2011

DOI : $10.4000 /$ ceg. 2011

ISSN : 2605-8359

Éditeur

Presses Universitaires de Provence

Édition imprimée

Date de publication : 15 juin 2014

Pagination : 117-134

ISSN : 0751-4239

Référence électronique

Jochen Mecke, «Une esthétique agonale de la Grande Guerre », Cahiers d'Études Germaniques [En

ligne], 66 | 2014, mis en ligne le 17 décembre 2017, consulté le 21 novembre 2020. URL : http://

journals.openedition.org/ceg/2011; DOI : https://doi.org/10.4000/ceg.2011 


\title{
Une esthétique agonale de la Grande Guerre
}

\author{
Jochen MECKE
}

Université de Regensburg

\section{La représentation de la guerre}

\section{Le conflit interculturel}

Qu'une littérature qui traite de la guerre et qui oppose deux groupes ennemis se construise sur une base agonale va sans dire. En effet, l'antagonisme entre les soldats de la Triple Entente d'une part et ceux des Empires Centraux de l'autre détermine la constellation des personnages et l'action de la plupart des romans et récits de guerre. Il se concrétise généralement dans un noyau narratif qui raconte l'affrontement des ennemis dans un combat armé. Ce qui peut surprendre quelquefois, par contre, c'est la violence du récit. Ainsi, Blaise Cendrars raconte dans J'ai tué (1918) comment il a pu égorger un soldat allemand dans la guerre de tranchées :

\footnotetext{
Me voici les nerfs tendus, les muscles bandés, prêt à bondir dans la réalité. J'ai bravé la torpille, le canon, les mines, le feu, les gaz, les mitrailleuses, toute la machinerie anonyme, démoniaque, systématique, aveugle. Je vais braver l'homme. Mon semblable. Un singe. Eil pour œil, dent pour dent. À nous deux maintenant. À coups de poing, à coups de couteau. Sans merci, je saute sur mon antagoniste. Je lui porte un coup terrible. La tête est presque décollée. J'ai tué le Boche. J'étais plus vif et plus rapide que lui. Plus direct. J'ai frappé le premier. J'ai le sens de la réalité, moi, poète. J'ai agi. J'ai tué. Comme celui qui veut vivre. $^{1}$
}

Il en est de même de l'autre côté du front : deux ans après Cendrars, Ernst Jünger décrit ses souvenirs de guerre dans In Stahlgewittern ${ }^{2}$.

\footnotetext{
${ }^{1}$ Blaise CENDRARS, « J'ai tué » [1918], in ID., Tout autour d'aujourd'hui, vol. 11, Paris, Denoel, 2002, p. 16.

${ }^{2}$ "In einer Mischung von Gefühlen, hervorgerufen durch Blutdurst, Wut und Alkoholgenuß gingen wir im Schritt auf die feindlichen Linien los. [...] Der übermächtige Wunsch zu töten, beflügelte meine Schritte. Der ungeheure Vernichtungswille, der über der Walstatt lastete, konzentrierte sich in den Gehirnen. [...] So mögen die Männer der Renaissance
} 
Les passages cités correspondent certainement aux attentes que peut avoir un lecteur quelconque à l'égard d'un récit de guerre. Néanmoins, ils sont plutôt exceptionnels car ils relatent ce combat entre deux individus avec une certaine brutalité, qui redouble la représentation de la violence par une violence de la représentation, tandis que dans la plupart des romans sur la Grande Guerre, il est rare d'être confronté à la description brutale d'un combat corps à corps. Ainsi, des auteurs comme Maurice Genevoix (Sous Verdun, 1916), Henri Barbusse (Le Feu, 1916), Roland Dorgelès (Les Croix de bois, 1919), Louis-Ferdinand Céline (Voyage au bout de la nuit, 1932), Ludwig Renn (Krieg, 1928), ou encore Erich Maria Remarque (Im Westen nichts Neues, 1929) - pour n'en citer que quelques-uns - ne racontent que rarement une confrontation directe entre deux individus. En revanche, beaucoup de ces romans représentent, outre le conflit opposant Anglais et Français d'un côté, et Allemands et Autrichiens de l'autre, d'autres conflits internes qui s'avèrent parfois plus importants que les premiers.

\section{Le conflit intraculturel}

Il est certain que la plupart des romans dépeignent, surtout au début de l'histoire, une ligne de démarcation claire et nette entre les parties ennemies. Mais au cours du récit, cette première frontière est complétée par une seconde, transversale, qui prend de plus en plus d'importance et finit presque par remplacer la première. À côté de la guerre au «front » s'ébauche un autre conflit qui oppose les simples soldats aux rangs supérieurs de la hiérarchie militaire. Déjà au début de son récit autobiographique La Main coupée, Blaise Cendrars raconte comment un officier et un sergent abandonnent leurs hommes aux rafales des mitrailleuses ennemies pour se mettre à l'abri du tir, ce qui oblige le caporal Cendrars à les faire arrêter ${ }^{3}$. Plus tard, Cendrars généralise sa critique des officiers et des généraux :

$$
\begin{aligned}
& \text { Ils [les officiers, J. M.] s'éclipsèrent tous, sauf qu'ils le firent avec beaucoup } \\
& \text { plus de souplesse que les sous-offs, ayant de meilleurs motifs et de plus hautes } \\
& \text { relations et beaucoup plus de bonnes raisons encore à invoquer sous prétexte de }
\end{aligned}
$$

von ihren Leidenschaften gepackt sein, so mag ein Cellini gerast haben, Werwölfe, die heulend durch die Nacht hetzen, um Blut zu trinken. [...] Da erblickte ich den ersten Feind. Eine Gestalt kauerte etwa drei Meter vor mir, anscheinend verwundet, in der Mitte der zertrommelten Mulde. Ich sah sie bei meinem Erscheinen zusammenfahren und mich mit weit geöffneten Augen anstarren, als ich ganz langsam, die Pistole vorstreckend, auf sie zuschritt. Zähneknirschend setzte ich die Mündung an die Schläfe des vor Angst Gelähmten [...] » (Ernst JüNGER, In Stahlgewittern. Aus dem Tagebuch eines Stoßtruppführers, Berlin, Mittler und Sohn, 3 édition 1922, p. 199). Au bout du compte, Jünger décide d'épargner le soldat allemand, ce qu'il ne fit point dans d'autres cas.

Un peu plus loin : « [...] Auch die Besatzung einer Reihe in einen Hohlweg eingebauter Unterstände stürzte heraus und entfloh. Ich schoß einen davon in dem Augenblick, als er aus dem Eingange des ersten sprang, nieder » (Ibid., p. 201).

3 Blaise CEnDRARS, La Main coupée [1946], Paris, Gallimard (coll. « Folio »), 1975, p. 84 sq., p. 92 . 
spécialité, de compétence, de cours de perfectionnement à suivre [...]. Dans la vie des tranchées ils étaient inexistants. ${ }^{4}$

Il en est de même pour les généraux qui «meurent dans leur lit, [comme] dit un proverbe canadien ${ }^{5} »$. En fait, ce qui oppose les officiers et les simples poilus, c'est ce qui devrait normalement les rapprocher, c'est-à-dire l'expérience partagée de la guerre : mais tandis que le quartier général est normalement situé plus loin de la ligne de combat, les soldats des tranchées sont directement exposés aux tirs des mitrailleuses, des canons, aux shrapnells ou aux obus et deviennent de véritables «chairs à canons »" Cet éloignement entraîne des «tirs amis » lorsque la ligne de démarcation semble passer, cette fois-ci, à l'intérieur des lignes françaises :

On s'était mis à nous tirer dessus. Pas les Allemands. Les Français ! On nous tirait dans le dos. Des feux de salve. Les copains. Les nôtres. Les escouades, demi-sections, sections, compagnies du régiment échelonnées derrière nous, probablement dans des positions aussi précaires que la nôtre, prenaient peur, s'affolaient.'

L'expérience de la guerre oppose également les soldats aux civils. Cela concerne en premier lieu les visiteurs du front. Barbusse peint un portrait assez critique des civils qui se comportent comme des touristes malsains et pervers sur le front, observant les poilus comme des animaux étranges.

\begin{abstract}
«-C'est les touristes des tranchées, dit à mi-voix Barque. [...] - Ah ! ah ! fait le premier monsieur, voilà des poilus... Ce sont de vrais poilus, en effet. Il s'approche un peu de notre groupe, un peu timidement, comme au Jardin d'Acclimatation, et tend la main à celui qui est le plus près de lui, non sans gaucherie, comme on présente un bout de pain à l'éléphant. - Hé, hé, ils boivent le café, fait-il remarquer. - On dit le « jus », rectifie l'homme-pie. - C'est bon, mes amis ? ${ }^{8}$
\end{abstract}

Les romans de guerre montrent également un autre type de conflit qui éclate quand les combattants sont en permission. Personne ne semble avoir relevé ce conflit d'une manière plus brutale que Cendrars dans La Femme et le Soldat, un récit décrivant de façon très abrupte la rencontre entre les soldats d'un régiment en permission et une jeune vendeuse de fruits ${ }^{9}$. Le comportement agressif des permissionnaires montre une véritable haine des soldats envers ceux qui n'ont pas connu l'horreur qu'ils ont vécue euxmêmes. Dans d'autres romans, les habitants des villages essaient de s'enrichir en vendant aux soldats leurs produits à des prix surfaits.

Ibid., p. 155 .

Ibid., p. 157.

Ibid..

${ }^{7}$ Ibid., p. 88.

${ }^{8}$ Henri BARBUSSE, Le Feu. Journal d'une escouade, Paris, Flammarion, 1916, p. 38.

9 Blaise CENDRARS, "La Femme et le soldat », in Tout autour d'aujourd'hui, t. VI, textes présentés et annotés par Michèle TOURET, Paris, Denoël, 2002. 


\section{Rapprochement des ennemis}

Mais si les soldats des tranchées s'éloignent de leurs généraux, officiers et sergents, ils se sentent en revanche parfois plus proches de leurs ennemis avec qui ils partagent l'expérience des tranchées. Ce que Christian Carion montre dans son film Joyeux Noël n'est pas une fiction cinématographique, mais un fait historique attesté ${ }^{10}$. Cependant, ce rapprochement entre les soldats de la Triple Entente et ceux des Empires centraux n'était pas limité à la Trêve de Noël, mais comportait également des avertissements aux soldats ennemis pour les prévenir d'un bombardement imminent ou bien des cessez-le-feu spontanés de plusieurs jours pour pouvoir enterrer les camarades morts ${ }^{11}$. Cet accord tacite avec l'ennemi existait également dans la vie quotidienne. Même Ernst Jünger, qui ne montre pourtant pas d'affection particulière pour les soldats anglais ou français, raconte ce genre de fraternisation dans In Stahlgewittern ${ }^{12}$.

Cette « entente cordiale » peut même se transformer en acte de solidarité et d'aide, comme Maurice Genevoix le raconte dans un épisode de Sous Verdun :
À la jumelle, je vois sur un chemin deux blessés qui se traînent, deux Français. Un des uhlans les a aperçus. Il a mis pied à terre et il s'avance vers eux. Je suis la scène de toute mon attention. Le voici qui les aborde, qui leur parle; et tous les trois se mettent en marche vers un gros buisson voisin de la route, l'Allemand entre les deux Français, les soutenant, les exhortant sans doute de la voix. Et là, précautionneusement, le grand cavalier gris aide les nôtres à s'étendre. Il est courbé vers eux, il ne se relève pas ; je suis certain qu'il les panse. ${ }^{13}$

Ainsi, dans plusieurs romans, au conflit externe s'ajoutent des conflits internes qui se jouent au sein d'une même culture, alors que s'installe une solidarité spontanée et inattendue entre les soldats ennemis. Le conflit intraculturel dont se double le conflit interculturel s'avère parfois plus important que ce dernier, ce qui rapproche les combattants ennemis tout en les éloignant de leurs supérieurs et des civils de leur propre culture. Mais la relation conflictuelle ne se manifeste pas seulement au niveau de l'histoire; elle détermine également le discours narratif.

${ }^{10}$ Christian CARION, Joyeux Noël, France, 2005

11 Jean-Jacques BECKER, L'Année 14, Paris, Armand Colin, 2005.

12 «Als ich am anderen Morgen völlig durchnäßt den Stollen verließ, glaubte ich meinen Augen nicht trauen zu dürfen. Das Gelände, dem bisher die Einsamkeit des Todes ihren Stempel aufgedrückt, hatte das Aussehen eines Jahrmarktes angenommen. Die Besatzung beider Gräben war von dem furchtbaren Schlamm auf die Brustwehren getrieben, und schon hatte sich vor den Drahtverhauen ein lebhafter Verkehr und Austausch von Schnaps, Zigaretten, Uniformknöpfen usw. entwickelt. Die Menge khakifarbener Gestalten, die den bisher so öden englischen Gräben entquoll, wirkte direkt verblüffend. » (JÜNGER, Stahlgewitter, p. 35).

${ }^{13}$ Maurice GenevolX, Sous Verdun, Août-octobre 1914, Paris, Hachette, 1916, p. 23. 


\section{Défense de la représentation de la mort}

Pour établir le lien entre la représentation du conflit et le conflit qui traverse cette représentation même, il est utile de reprendre l'épisode déjà cité de « J'ai tué » de Blaise Cendrars. En réalité, la singularité de ce texte apparaît d'une manière encore plus nette, si nous le comparons à une version ultérieure de Cendrars dans son récit autobiographique La Main coupée en 1946 :

C'est durant ce nettoyage [i.e. des tranchées, J. M.] que j'ai tué d'un coup de couteau un Allemand qui était déjà mort. Il me guettait, embusqué derrière un pare-éclats, le fusil en arrêt. Je lui sautai dessus et lui portai un coup terrible qui lui décolla presque la tête et qui le fit tomber à la renverse, semant son casque à pointe. Alors, je constatai qu'il était déjà mort depuis le matin et qu'il avait eu le ventre ouvert par un obus. Il s'était vidé. Jamais un homme ne m'a fait aussi peur. $^{14}$

En insistant sur le fait que c'est un soldat déjà mort à qui le narrateur autodiégétique coupe la gorge, l'acte de tuer est révoqué, comme si Cendrars avait, des années plus tard, senti le besoin d'amortir le choc de son premier récit. Cette révocation est d'autant plus surprenante qu'elle s'opère dans un contexte qui, en soi, était tout à fait propice à une surenchère de la violence narrative. Car La Main coupée a été écrit après la Seconde Guerre mondiale et par conséquent dans un sentiment de haine à l'encontre de l'Allemagne, un pays responsable non seulement d'une guerre atroce et de l'holocauste, mais également de la mort du fils de Cendrars, tué en Afrique du Nord comme pilote de guerre dans un accident d'avion ${ }^{15}$. Compte tenu de ce contexte, il est étonnant que le même Cendrars - qui par ailleurs traite les Allemands de tous les noms et se complaît à raconter comment ses camarades et lui avaient, avec succès, truffé un gramophone d'explosifs pour attirer et tuer un maximum de soldats allemands - ait senti le besoin d'atténuer la violence de la première version de $1918^{16}$.

Dans Sous Verdun, Maurice Genevoix fait montre des mêmes réticences. Dans la première édition, Genevois décrit comment il s'est soudain retrouvé entouré d'Allemands qui étaient en train de passer à l'attaque contre les lignes françaises. Dans cette situation absurde, il ne lui reste rien d'autre que de hurler avec les loups au sens propre du terme pour ne pas se faire reconnaître. Comme il fait nuit et qu'il peut se coiffer d'un casque allemand trouvé par hasard, il réussit à passer inaperçu et à courir avec les Allemands jusqu'au moment où ceux-ci doivent se reformer avant de passer au prochain assaut. À la suite de ce passage, Genevois écrit :

Déjà il n'y a plus de braillards à voix rauque [i.e. d'Allemands, J. M.]. [...] Pourtant avant de rallier les chasseurs, $j$ 'ai rattrapé encore trois fantassins

\footnotetext{
${ }^{14}$ CEndRARS, La Main coupée, p. 120.

${ }^{15}$ Ibid., p. 11 sq.

${ }^{16}$ Ibid., p. 169 sq.
} 
allemands isolés. Et à chacun, courant derrière lui du même pas, j'ai tiré une balle de revolver dans la tête ou dans le dos. Ils se sont effondrés avec le même cri étranglé. ${ }^{17}$

Il est ici intéressant de noter que Genevoix avait senti le besoin de supprimer cette scène à la première réimpression de son livre. Il commente cette autocensure de la manière suivante :

Lors d'une réimpression du livre j'avais supprimé ce passage : c'est une indication quant à ces retours sur soi-même que devaient fatalement se produire. Je la rétablis aujourd'hui, tenant pour un manque d'honnêteté l'omission volontaire d'un des épisodes de guerre qui m'ont le plus profondément secoué et qui ont marqué ma mémoire d'une empreinte jamais effacée. (Note de 1949) ${ }^{18}$.

Les mêmes réticences à l'égard du récit de mise à mort sont à l'œuvre dans Le Feu de Barbusse. S'il raconte comment ses camarades ont tué un Allemand, la manière dont il évoque cet épisode est néanmoins symptomatique à plusieurs égards. Le motif qui déclenche toute l'action est insignifiant : il manque des allumettes aux soldats français pour préparer le repas. À la recherche d'une boîte d'allumettes, ils s'égarent dans les tranchées allemandes :

\begin{abstract}
- Des chargeurs ! dit à voix basse Pépin... Des chargeurs boches sur la planchette ! Nous sommes dans le boyau boche !-Mettons-les. Il y a un élan des trois hommes pour sortir. - Attention, bon Dieu ! Bougez pas !... Les pas... On entend marcher. C'est le pas assez rapide d'un homme seul. Ils ne bougent pas, retiennent leur souffle. Leurs yeux braqués à ras de terre voient la nuit remuer, à droite, puis une ombre avec des jambes, se détache, approche, passe... Cette ombre se silhouette. Elle est surmontée d'un casque recouvert d'une housse sous laquelle on devine la pointe. Aucun autre bruit que celui de la marche de ce passant. À peine l'Allemand est-il passé que les quatre cuisiniers, d'un seul mouvement, sans s'être concertés, s'élancent, se bousculent, courent comme des fous, et se jettent sur lui. - Kamerad, messieurs ! dit-il. Mais on voit briller et disparaître la lame d'un couteau. L'homme s'affaisse comme s'il s'enfonçait par terre. Pépin saisit le casque tandis qu'il tombe et le garde dans sa main. ${ }^{19}$
\end{abstract}

Si la scène décrit bien l'assassinat du soldat allemand par les quatre poilus français, Barbusse évite toutefois de nommer l'auteur grâce à une ellipse narrative qui évoque seulement la lame de couteau qui brille un moment et disparaît ensuite, comme si le couteau avait agi tout seul. La scène est imprégnée de toutes ces ellipses, suppressions et euphémismes qui permettent d'éluder une description trop directe de la mise à mort. Cela est nécessaire parce que, comme l'acte de tuer en lui-même, la représentation de l'acte de tuer a besoin d'une légitimation. Mais tandis que la guerre donne le droit de tuer, le récit de guerre ne donne pas automatiquement la licence poétique de

${ }^{17}$ GenevolX, Sous Verdun, p. 51.

18 Ibid.,

${ }^{19}$ BARBusse, Le Feu, p. 234. 
représenter les meurtres commis par les soldats. Les réticences de Barbusse, de Cendrars et de Genevoix révèlent l'existence d'un conflit important entre éthique et esthétique qui se situe au sein de l'écriture même.

\section{Hommes et machines}

Que ce conflit entre esthétique et éthique puisse prendre une telle importance relève d'une forme nouvelle et particulière de guerre : la guerre à distance. Car dans la mesure où il constitue une exception, l'acte de tuer un ennemi dans un combat corps à corps acquiert une importance éthique plus grande. Si l'on examine le texte de Cendrars de plus près, on peut y détecter un motif de la haine inouïe éprouvée par le protagoniste. En effet, c'est la haine de « toute cette machinerie démoniaque systématique et aveugle ${ }^{20}$ qui se décharge par métonymie dans l'attaque contre le soldat allemand. La plupart des romans de la Grande Guerre tiennent compte du changement radical provoqué par les nouveaux dispositifs techniques mis en place, pour la première fois, de manière si massive. Ce qui - au moins encore dans les guerres précédentes - pouvait être considéré comme un adversaire humain se voit transformé en pure cible technique, tandis que les machines de guerre évoquées dans $J$ 'ai tué deviennent les véritables adversaires. Cela est particulièrement visible dans les scènes de combat à distance où la grande portée des mitrailleuses, grenades, obus et fusées fait disparaître les ennemis au profit d'une machinerie militaire anonyme qui peut frapper à tout moment. Le résultat de ce changement ne saurait être plus radical : le combat de l'homme contre l'homme est remplacé par une guerre entre hommes et machines ou, pire encore, par le combat de la machine contre la machine, canon contre canon et fusée contre fusée, qui ne laisse plus guère de place à l'homme.

C'est Jünger lui-même qui décrit dans les premiers paragraphes de In Stahlgewittern les effets de cette nouvelle forme de guerre à distance sans ennemi personnel visible ${ }^{21}$. Il en est de même pour Roland Dorgelès qui décrit un bombardement dans Les Croix de bois :

\footnotetext{
${ }^{20}$ CENDRARS, « J'ai tué », p. 16.

${ }^{21}$ « Plötzlich dröhnte eine Reihe dumpfer Erschütterungen in der Nähe, während aus allen Häusern Soldaten dem Dorfeingang zustürzten. Wir befolgten dies Beispiel, ohne recht zu wissen warum. Wieder ertönte ein eigenartiges, nie gehörtes Flattern und Rauschen über uns und ertrank in polterndem Krachen. [...] Gleich darauf erschienen schwarze Gruppen auf der menschenleeren Dorfstraße, in Zeltbahnen oder auf den verschränkten Händen schwarze Bündel schleppend. Mit einem merkwürdig beklommenen Gefühl der Unwirklichkeit starrte ich auf eine blutüberströmte Gestalt mit lose am Körper herabhängendem Bein, die unaufhörlich ein heiseres »Zu Hilfe!« hervorstieß [...]. Das war so rätselhaft, so unpersönlich. [...] Kaum, daß man dabei an den Feind dachte, dieses geheimnisvolle, tückische Wesen irgendwo dahinten. Das völlig außerhalb der Erfahrung liegende Ereignis machte einen so starken Eindruck, daß es Mühe kostete, die Zusammenhänge zu begreifen. » (JÜNGER, In Stahlgewittern, p. 2).
} 


\begin{abstract}
Brusquement, une rafale d'explosions les secoua. Ce fut une seconde d'affolement. Ils se levaient, sortaient des trous, se bousculaient pour prendre leurs fusils, tout de suite hébétés par le tonnerre assourdissant de l'artillerie subitement déchaîné. Au même signal, sur toute la ligne, nos pièces s'étaient mises à tirer, et dans ce déchirant fracas, on n'entendait même plus les obus rayer l'air. Nous nous étions précipités aux créneaux, fouillant déjà la cartouchière. Au bout du terrain vague qui séparait les deux réseaux, juste sur la ligne allemande dont on apercevait encore le lacet sinueux dans la fumée, les obus tapaient à coups furieux, faisant voler des morceaux de tranchée blanche, comme des copeaux sous un rabot de menuisier. Enervés, nous courions de droite à gauche, on s'appelait, on se renseignait l'un l'autre, sans rien savoir. ${ }^{22}$
\end{abstract}

La plupart des romans de 1914-18 décrivent longuement des scènes de bombardement à distance de ce type, sans que jamais un ennemi apparaisse. Tous les soldats sont réduits à subir l'attaque de la machine de guerre adverse, tout en restant passifs, comme s'il s'agissait d'une intempérie. En ce sens, le titre In Stahlgewittern est bien choisi, car les possibilités pour l'individu de combattre des canons, des obus, des mitrailleuses et d'autres armes à distance sont aussi réduites que le seraient celles d'humains voulant combattre des orages. Ainsi, l'ennemi reste invisible ${ }^{23}$.

M. Genevoix insiste également sur la rareté du combat avec un ennemi visible. Quand il raconte comment il a réussi à tuer des soldats allemands qui se trouvaient près de lui, il précise que " ç'a été la seule occasion - la seconde et la dernière a été aux Éparges, le 18 février au matin - où j'ai senti en tant que telles la présence et la vie des hommes sur qui je tirais ${ }^{24} \gg$.

Cette nouvelle forme de guerre, la guerre des machines, ne permet plus de conserver la continuité de l'action. Ainsi, Genevoix, lorsqu'il raconte dans le premier chapitre la «prise de contact » avec l'ennemi, utilise un oxymore puisque cette prise de contact se fait à distance. Dès lors, le récit ne peut raconter le combat qu'en se limitant à constater les dégâts causés par les canons et les obus allemands :

Soudain, un sifflement rapide qui grandit, grandit... et voilà deux shrapnells qui éclatent, presque sur ma tranchée. Je me suis baissé ; j'ai remarqué surtout l'expression angoissée d'un de mes hommes. Cette vision me reste. Elle fixe mon impression. ${ }^{25}$

\title{
Une modification radicale du récit de guerre
}

Cette nouvelle forme de guerre ne peut plus être racontée selon les formes du récit héritées de la tradition littéraire. Elle entraîne une modification

${ }^{22}$ Roland Dorgelès, Les Croix de bois, Paris, Albin Michel, 1964, p. 46.

23 "Die Schlacht von "Les Eparges" war meine erste. Sie war ganz anders, als ich gedacht. Ich hatte an einer großen Kampfhandlung teilgenommen, ohne einen Gegner zu Gesicht bekommen zu haben » (JÜNGER, In Stahlgewittern, p. 20).

${ }_{24}^{24}$ Maurice GeNEVOIX, Ceux de 14, Paris, Flammarion, rééd. 2007, p. 51.

${ }^{25}$ ID., Sous Verdun, p. 20 sq. 
narrative considérable : la machinerie militaire anonyme destitue l'homme de son rôle traditionnel de sujet de l'action pour prendre définitivement sa place et révolutionner, de ce fait, la relation entre homme et machine. Le dispositif technique est promu au rang de véritable actant d'une guerre qui assigne à l'homme un rôle subalterne :

\begin{abstract}
Ligne de section par quatre dans le bois, près d'une clairière. Les chaudrons dégringolent ; un réserviste, grand, blond roux, au premier qui explose, se retourne brusquement, me crie qu'il est blessé. Il est blafard et il tremble violemment : c'est une branchette qui l'a piqué, comme il se baissait. Un second chaudron, et c'est la ruée fantastique de Ferrai, serrant son poignet ensanglanté. Un troisième : le caporal Trémault reçoit dans la joue le bout d'un canon de fusil. Il est estomaqué un moment, puis, ses esprits revenus, il sacre jusqu'à extinction. L'arrosage continue. ${ }^{26}$
\end{abstract}

La structure traditionnelle du récit et de la phrase ne peut plus être conforme à une guerre menée par des machines. La séquence sujet-verbe est remplacée par un style télégraphique. Les ellipses du verbe permettent de rendre la forme particulière du nouvel art de la guerre à distance, qui contraint l'homme à accuser les coups assénés par les armes de longue portée. La plupart des particularités narratives des récits de 1914-18 découlent de ce nouvel art de la guerre, fait d'armes de destruction massive, de batailles à distance et de guerres de tranchées immobiles sans individus.

\title{
Dimensions esthétiques de la guerre
}

Cependant, les romans de 14-18 décrivent souvent la beauté éblouissante de cette nouvelle forme de guerre. Dans In Stahlgewittern, Jünger évoque à plusieurs reprises cette splendeur du spectacle des fusées et obus ${ }^{27}$. Dorgelès décrit la même beauté insolite du spectacle : «La nuit, c'était là qu'on tirait le feu d'artifice : globes rouges, étoiles blanches, chenilles vertes balancées, vision splendide des nuits de guerre. Des éclairs d'éclatements y joignaient leur fracas ${ }^{28}$. " Cette beauté déconcertante de la nouvelle guerre ne manque pas de provoquer de l'admiration chez les soldats. Chez Barbusse, l'escouade du narrateur est littéralement subjuguée :

[...] Tout à coup une étoile intense s'épanouit là-bas, vers les lieux vagues où nous allons : une fusée. Elle éclaire toute une portion du firmament de son halo

${ }^{26}$ Ibid., p. 21.

27 « Mit jener eigentümlichen Mißstimmung, die eine in der Morgensonne strahlende Landschaft auf die erschöpften, übernächtigten Nerven ausübt, zogen wie durch die Laufgräben nach Monchy und von dort zu der sich vor dem Waldrande von Adinfer hinziehenden zweiten Stellung, von wo wir einen grandiosen Ausblick auf den ersten Auftakt zur Sommeschlacht hatten. Die Frontabschnitte links von uns waren in weiße und schwarze Rauchwolken gehüllt, turmhoch spritzte ein schwerer Einschlag neben dem anderen; darüber zuckten zu Hunderten die kurzen Blitze platzender Schrapnells. » (JÜNGER, In Stahlgewittern, p. 54).

${ }^{28}$ DORGELÈs, Les Croix de bois, p. 159 sq. 


\begin{abstract}
laiteux, en effaçant les constellations, et elle descend gracieusement avec des airs de fée. Une rapide lumière en face de nous, là-bas ; un éclair, une détonation. C'est un obus. [...] L'obus est tombé sur le sommet, dans nos lignes. Ce sont eux qui tirent. Un autre obus. Un autre, un autre, plantent, vers le haut de la colline, des arbres de lumière violacée dont chacun illumine sourdement tout l'horizon. Et bientôt, il y a un scintillement d'étoiles éclatantes et une forêt subite de panaches phosphorescents sur la colline : un mirage de féerie bleu et blanc se suspend légèrement à nos yeux dans le gouffre entier de la nuit. [...]

- C'est comme si tu vois un feu d'artifice, disent-ils. [...] voici une étoile rouge, une verte; une gerbe rouge, beaucoup plus lente. [...] Ce sont les Allemands qui font des signaux, et aussi les nôtres qui demandent de l'artillerie. ${ }^{29}$
\end{abstract}

Dans Les Croix de bois, les soldats éprouvent la même admiration :

Pas bien loin, les fusées barraient la nuit d'un long boulevard de clarté, et, par instants, cela s'égayait de lueurs rouges ou vertes, vite éteintes, pareilles à des enseignes lumineuses. Ce ciel de guerre faisait penser à une nuit populaire de quatorze juillet. (Dorgelès, p. 39). Quand une salve bien pointée donnait sur la tranchée ses quatre coups de pic, arrachant une gerbe de terre, de pierres et de madriers, un cri d'admiration montait, une clameur ravie de feu d'artifice. Dans le vacarme, on n'entendait plus que ce rire heureux, ce rire honnête, comme si nous avions jugé l'effet de balles à massacre, sur les têtes de bois d'une noce villageoise. $^{30}$

Mais c'est justement cet aspect esthétique qui pose un problème fondamental pour tous les romans sur la guerre de 14-18. Car la «beauté » de ce feu d'artifice, si elle constitue un spectacle impressionnant pour les soldats, cause également la mort de centaines d'entre eux. En ce sens, la scène topique des récits de la Grande Guerre, dans laquelle le bombardement est perçu comme un feu d'artifice, ne concerne pas seulement le droit de décrire l'aspect esthétique d'un bombardement, mais aussi la légitimité de toute esthétique de la guerre.

Ce sont évidemment les futuristes italiens qui les premiers ont magnifié la beauté de la guerre. Déjà dans le premier manifeste futuriste, publié dans $L e$ Figaro en 1909, Marinetti célèbre la guerre : « Nous voulons glorifier la guerre - seule hygiène du monde -, le militarisme, le patriotisme, le geste destructeur des anarchistes, les belles idées qui tuent, et le mépris de la femme ${ }^{31} »$. Ni la Première Guerre mondiale, à laquelle Marinetti a participé lui-même, ni la mort de plusieurs futuristes n'ont pu le faire revenir sur ses positions. En 1935, il réitère sa célébration de la guerre dans le manifeste intitulé « Invito a la guerra africana ${ }^{32} »$.

C'est pourquoi [...] nous affirmons ceci : la guerre est belle, parce que, grâce aux masques à gaz, au terrifiant mégaphone, aux lance-flammes et aux petits chars

${ }^{29}$ BARBuSSE, Le Feu, p. 224.

DORGELÈs, Les Croix de bois, p. 47.

${ }^{11}$ Filippo Tomaso MARINETTI, « Manifeste futuriste », Le Figaro, 20 février 1909, p. 1.

32 ID., "Invito alla guerra africana. Manifesto agli scrittori e agli artisti d'Italia », $L a$ Gazzetta del Popolo, Torino, 30 juillet 1935, p. 3. 


\begin{abstract}
d'assaut, elle fonde la souveraineté de l'homme sur la machine subjuguée. La guerre est belle, parce qu'elle réalise pour la première fois le rêve d'un homme au corps métallique. La guerre est belle, parce qu'elle enrichit un pré en fleurs des orchidées flamboyantes que sont les mitrailleuses. La guerre est belle, parce qu'elle rassemble, pour en faire une symphonie, la fusillade, les canonnades, les suspensions de tir, les parfums et les odeurs de décomposition. La guerre est belle, parce qu'elle crée de nouvelles architectures, comme celle des grands chars, des escadres aériennes aux formes géométriques, des spirales de fumée montant des villages incendiés, et bien d'autres encore (...). Écrivains et artistes futuristes, $[\ldots]$ rappelez-vous ces principes fondamentaux d'une esthétique de guerre, pour que soit ainsi éclairé [...) votre combat pour une nouvelle poésie et une nouvelle sculpture $!^{33}$
\end{abstract}

Dans la postface de son essai, Das Kunstwerk im Zeitalter seiner technischen Reproduzierbarkeit, Walter Benjamin avance sa célèbre hypothèse selon laquelle le fascisme promeut l'esthétisation de la politique ${ }^{34}$. Selon lui, celle-ci aurait pour fonction principale de limiter l'action des masses, qui veulent le changement politique, à sa seule représentation dans des manifestations esthétisantes. Ainsi, la manifestation fasciste empêche l'action, elle se complaît dans l'expérience dionysiaque de l'ivresse, sans aboutir à une conséquence pratique quelconque. Voilà pourquoi, pour Benjamin, l'esthétisation de la politique trouve son aboutissement ou son point culminant dans la guerre. Selon lui, la déshumanisation de la guerre et de l'être humain signifie également une révolte de la technique contre l'homme, une technique qui doit récupérer auprès du matériel humain ce que la société lui a soustrait comme matériel naturel. Ainsi, au lieu de canaliser des fleuves, la technique canalise désormais des flots de sang humain vers les tranchées. Dans cette perspective, les batailles de matériel apparaissent comme l'effet d'une technique déchaînée qui se retourne contre l'homme. La révolte des machines prend l'homme pour cible ${ }^{35}$.

Ce phénomène d'esthétisation ne concerne pas seulement la politique telle qu'elle est conçue par le fascisme, mais aussi la guerre elle-même. Si les soldats peuvent admirer le spectacle insolite de la beauté de la guerre, cela est justement dû aux nouvelles armes qui permettent d'attaquer l'ennemi à longue distance. C'est cet éloignement qui donne au combattant une position d'observateur qui lui permet de prendre du recul par rapport aux événements, recul qui est la condition de la transformation du combat en spectacle et de la naissance d'un regard esthétique. Tel est par exemple le cas lorsque les

33 Ibid.

34 Walter BENJAMIN, Das Kunstwerk im Zeitalter seiner technischen Reproduzierbarkeit, Frankfurt/M., Suhrkamp, 1963, p. 48-51.

35 " Der imperialistische Krieg ist ein Aufstand der Technik, die am „Menschenmaterial“ die Ansprüche eintreibt, denen die Gesellschaft ihr natürliches Material entzogen hat. Anstatt Flüsse zu kanalisieren, lenkt sie den Menschenstrom in das Bett ihrer Schützengräben, anstatt Saaten aus ihren Aeroplanen zu streuen, streut sie Brandbomben über die Städte hin, und im Gaskrieg hat sie ein Mittel gefunden, die Aura auf neue Art abzuschaffen. » (Ibid., p. 50). 
soldats de l'escadron du narrateur du $\mathrm{Feu}$ de Barbusse admirent les fusées et les signaux des deux armées comme un feu d'artifice.

Bien sûr, cette dimension esthétique de la guerre n'est pas un phénomène entièrement nouveau. Les guerres précédentes faisaient déjà montre d'une certaine esthétisation. L'organisation de l'armée, les couleurs des drapeaux et des uniformes, ainsi que le prestige de ces derniers auprès des citoyens et des femmes, les défilés, la marche au pas cadencé, les chansons des armées, les cris lors de l'attaque, les formations militaires avant celle-ci, tout cela constitue un dispositif qui dote également la guerre traditionnelle d'une dimension esthétique.

La littérature a également contribué à cette esthétisation. De nombreux textes ont participé à la création d'un discours esthétique sur la guerre qui est doté de différents éléments constitutifs : le héros unique et individualisé qui vainc son adversaire ou meurt de façon héroïque lors d'un combat, les actes de bravoure des héros de guerre qui acquièrent gloire et renommée par leur mort et leur sacrifice pour la nation, et la guerre comme possibilité de transcender l'homme dans une expérience dionysiaque où se mélangent les individus. Tous ces éléments ont créé un discours qui a depuis toujours exercé une certaine fascination sur les peuples.

Mais si la littérature, depuis l'Iliade d'Homère, a toujours embelli la guerre, cette esthétisation prend une forme particulière dans les œuvres littéraires qui précèdent la Première Guerre Mondiale, avec des auteurs tels que Maurice Barrès, Léon Bloy, Paul Déroulède ou bien Ernest Psichari. Dans L'Appel des armes (1913) par exemple, Psichari réunit tous les éléments nécessaires à la préparation esthétique de la guerre, comme par exemple la force unificatrice et purifiante de l'armée, l'expérience héroïque du combat et une valorisation esthétique de la machinerie de guerre se composant de « beaux canons, précis et gais, avec des ornements qui n'en sont pas [...] (de) beaux joujoux [...] l'absolue perfection [...] d'un beau travail métallurgique $^{36} »$. Pour lui, rien ne semble plus digne d'admiration que le chef qui se dresse devant l'ennemi la poitrine nue, sabre au clair.

À toute cette fascination littéraire pour la guerre s'ajoutent d'autres éléments importants relatifs à la jeunesse de 1914, comme le montre l'exemple d'Ernst Jünger ${ }^{37}$. Le jeune Jünger attend de la guerre de 14-18 une expérience esthétique où l'enivrement et l'extase se joignent pour l'arracher à la vie quotidienne normale et dont il a décrit les enjeux dans son essai «Der

${ }^{36}$ Ernest Psichari, L'appel des armes, Paris, Oudin, 1913, p. 131.

37 « Wir hatten Hörsäle, Schulbänke und Werktische verlassen und waren in den kurzen Ausbildungswochen zusammengeschmolzen zu einem großen, begeisterten Körper, Träger des deutschen Idealismus der nachsiebziger Jahre. Aufgewachsen im Geiste einer materialistischen Zeit, wob in uns allen die Sehnsucht nach dem Ungewöhnlichen, nach dem großen Erleben. Da hatte uns der Krieg gepackt wie ein Rausch. In einem Regen von Blumen waren wir hinausgezogen in trunkener Morituri-Stimmung. Der Krieg mußte es uns ja bringen, das Große, Starke, Feierliche. Er schien uns männliche Tat, ein fröhliches Schützengefecht auf blumigen, blutbetauten Wiesen. Kein schönrer Tod ist auf der Welt... Ach, nur nicht zu Haus bleiben, nur mitmachen dürfen! » (JÜNGER, In Stahlgewittern, p. 1). 
Kampf als inneres Erlebnis ». Mais déjà, dans In Stahlgewittern, il célèbre le danger de la mort ${ }^{38}$. Cette attitude de Jünger était loin d'être un cas unique d'enthousiasme, comme l'ont démontré les études sur les attitudes des intellectuels avant la Première Guerre mondiale ${ }^{39}$.

\section{Critique de l'esthétisation}

Dans l'un des romans les plus curieux sur la Première Guerre mondiale, Thomas l'imposteur (1923), Jean Cocteau propose une analyse pertinente de ce désir d'expérience esthétique. Il y raconte l'histoire de la princesse de Bormes qui veut organiser avec l'aide de quelques amis - entre autres Thomas Fontenoy - des transports de blessés de guerre. Cependant, le motif pour lequel elle et Thomas veulent aider les blessés n'est point la charité ou l'amour du prochain, mais le besoin d'éprouver des sensations fortes : «Elle voulait s'amuser et savait s'amuser ${ }^{40} »$. Ainsi, « la guerre lui apparut tout de suite comme le théâtre de la guerre. Théâtre réservé aux hommes ${ }^{41}$. " Elle développe une véritable soif de guerre, parce que la mode d'alors est au danger ${ }^{42}$. Pour la princesse qui « sentait la gloire comme un cheval l'écurie ${ }^{43}{ }^{\prime}$, tout se transforme immédiatement en spectacle. Ainsi, à la vue des champs de bataille et de quelques dragons, elle imagine se trouver dans les coulisses d'un théâtre et perçoit les soldats comme des figurants qui s'habillent $^{44}$. Il ne saurait y avoir d'analyse plus sarcastique des motivations bellicistes fondées principalement sur des besoins esthétiques. Certes, l'on peut objecter que l'envergure de la critique de Cocteau est limitée, car elle ne s'applique qu'à ceux qui sont condamnés à être les simples observateurs d'une guerre dont ils sont exclus. Mais lors du bombardement de Reims, la princesse et Thomas participent bel et bien aux affrontements et, à la fin du roman, Thomas devient un véritable soldat. Il entre tellement bien dans son rôle qu'il se laisse prendre à son propre jeu ${ }^{45}$, si bien que, lorsqu'il doit apporter personnellement un message à un autre poste au front, rôle et réalité se confondent. Surpris par une patrouille allemande, il provoque ses

\footnotetext{
38 «Die Gefahr ist der vornehmste Augenblick seines Berufes, da gilt es, gesteigerte Männlichkeit zu beweisen. Ehre und Ritterlichkeit erheben ihn zum Herrn der Stunde. Was ist erhabener, als hundert Männern voranzuschreiten in den Tod? Gefolgschaft wird solcher Persönlichkeit nie versagt, die mutige Tat fliegt wie Rausch durch die Reihen. » (Jünger, In Stahlgewittern, p. 18).

${ }^{39}$ Helmut FRIES, Die große Katharsis. Der Erste Weltkrieg in der Sicht deutscher Dichter und Gelehrter, vol. 1, Die Kriegsbegeisterung von 1914: Ursprünge - Denkweisen - Auflösung, Konstanz, Verlag am Hockgraben, 1994.

${ }^{40}$ Jean COCTEAU, Thomas l'imposteur, Paris, Gallimard, rééd. 2010, p. 12.

${ }^{41}$ Ibid., p. 16.

${ }^{42}$ Ibid., p. 17.

${ }^{43}$ Ibid., p. 19

${ }^{44}$ Ibid., p. 37.

${ }^{45}$ Ibid., p. 27.
} 
adversaires et prend ensuite la fuite, mais les Allemands tirent sur lui et l'atteignent à la poitrine :

Il tomba. Il devenait sourd. «Une balle se dit-il. Je suis perdu si je ne fais pas semblant d'être mort. » Mais, en lui, la fiction et la réalité ne formaient qu'un. Guillaume Thomas était mort. ${ }^{46}$

Ainsi, le roman de Cocteau conjugue la fiction esthétique avec la réalité de la guerre pour former un ensemble inextricable. Tout en montrant les motivations personnelles de ses personnages - participer à la guerre pour des motifs esthétiques -, il les dénonce et arrive à analyser le mécanisme qui a incité des jeunes gens à s'engager comme volontaires.

À l'instar de Thomas l'imposteur, beaucoup de romans de 14-18 rejettent le discours esthétisant sur la guerre et les manifestations littéraires qui en résultent. Ce n'est pas un hasard si Genevoix relate dans son récit daté du vendredi 28 août le discours pathétique d'un commandant qui cite Déroulède : «Et là, un commandant à monocle nous lit, d'une voix sèche, une proclamation vibrante : oraison funèbre du colonel, exhortations véhémentes, vers de Déroulède pour finir ${ }^{47}$. " Dans Les Croix de bois, Dorgelès raconte les réactions des soldats face à l'ordre d'attaquer :

Les camarades non plus ne rient pas : rien qu'en tournant la tête, ils pourraient voir, par le créneau, ceux de la dernière attaque, restés couchés dans l'herbe haute. Aucun ne sort un médaillon de sa poche pour l'embrasser furtivement, aucun non plus ne s'écrie, comme dans les contes : «Enfin ! On va sortir des trous. $)^{48}$

Évidemment, en évoquant le baiser posé sur le médaillon et les cris de joie, le texte prend le contre-pied de toute une littérature de guerre néoromantique ou enthousiaste à la manière de Psichari, qualifiée ici de conte de fée. Mais le rejet le plus explicite de cette littérature se trouve probablement dans La Main coupée, lorsque Cendrars reçoit la visite d'un agent secret - qui est également un écrivain peu talentueux s'enthousiasmant pour la guerre, qu'il considère comme une source d'inspiration esthétique :

La guerre, pontifiait l'autre, mais c'est sublime. Je suis en train d'écrire le plus beau poème de ma vie. Vous allez pas me faire croire, Blaise Cendrars, que tout ce qui se passe au front ne vous inspire pas ? - Oh, pas du tout. - Vous devez avoir des poèmes plein la poche. - Pas un! Alors, pourquoi vous êtes-vous engagé ? En tout cas pas pour tenir un porte-plume. [...] - J'entends, mais c'est existant ! Je croyais, qu'avec votre amour de la vie, Blaise Cendrars, la vie pleine que vous menez ici, le danger, la camaraderie, le décor camouflé, la nature, le plein air, le rythme industriel dans lequel on est plongé dès qu'on pénètre dans les tranchées, le pittoresque, l'imprévu, les rencontres surprenantes,

${ }^{46}$ Ibid, p. 130.

${ }^{47}$ GenEVoiX, Sous Verdun, p. 16.

48 Dorgelès, Les Croix de bois, p. 85. 
la mort violente, je croyais qu'avec la guerre vous étiez dans votre élément, et que tout cela vous inspirerait, Blaise Cendrars. ${ }^{49}$

Le discours de l'agent-écrivain reprend les stéréotypes de toute une littérature qui peint un tableau idéalisé d'une guerre propice à l'esthétique, que Cendrars rejette violemment dans sa réponse tout en faisant allusion au discours esthétisant sur la guerre :

\begin{abstract}
- « Mourir pour la patrie est le sort le plus beau... » n'est-ce pas ? Vous croyezvous au théâtre, Monsieur? Avez-vous perdu le sens de la réalité ? Vous n'êtes pas au Français, ici. Et savez-vous ce qui se cache sous cet alexandrin ? La guerre est une ignominie. Tout au plus ce spectacle peut satisfaire les yeux, le cœur d'un philosophe cynique et combler la logique du pessimisme le plus noir. La vie dangereuse peut convenir à un individu, certes, mais sur le plan social, cela mène directement à la tyrannie $[\ldots]^{50}$
\end{abstract}

La réponse de Cendrars est dans le droit fil de l'expérience qu'il a vécue pendant la guerre et qui s'avère une désillusion dont l'engagé volontaire fait les frais : il constate que " ces souffrances étaient sans grandeur » et que «tout cela manquait de gloire $»^{51}$, et cela est valable pour beaucoup de personnages dans les romans de la Grande Guerre. Dans ces conditions, les possibilités de devenir un héros sont de fait limitées. « Nous étions remontés en ligne devant Herbécourt, dans la tranchée Clara, où tout l'héroïsme consistait de résister durant quatre jours à la succion de la boue qui faisait ventouse par en bas $^{52} »$. Les personnages de Dorgelès partagent la même déception à l'égard de la guerre de tranchées qui ne se conforme aucunement à leurs attentes :

C'était pour lui une déception, cette première vision de la guerre. Il aurait voulu être ému, éprouver quelque chose, et il regardait obstinément vers les tranchées, pour se donner une émotion, pour frissonner un peu. Mais il se répétait : "C'est la guerre... Je vois la guerre » sans parvenir à s'émouvoir. Il ne ressentait rien, qu'un peu de surprise. Cela lui semblait tout drôle et déplacé, cette féerie électrique au milieu des champs muets. Les quelques coups de fusil qui claquaient avaient un air inoffensif. Même ce village dévasté ne le troublait pas : cela ressemblait trop à un décor. C'était trop ce qu'on pouvait imaginer. Il eût fallu des cris, du tumulte, une fusillade, pour animer tout cela, donner une âme aux choses : mais cette nuit, ce grand silence, ce n'était pas la guerre... Et c'était bien elle pourtant : une rude et triste veille plutôt qu'une bataille. ${ }^{53}$

\footnotetext{
${ }^{49}$ CEndRARS, La Main coupée, p. 378.

${ }^{50}$ Ibid.

${ }^{51}$ Ibid., p. 215 sq.

${ }^{52}$ Ibid., p. 216

${ }^{53}$ DORGELÈs, Les Croix de bois, p. 41.
} 


\section{La guerre contre l'esthétique}

Les déceptions et les critiques évoquées par Dorgelès et Cendrars ne sont pas des prises de position isolées. Elles constituent plutôt une mise en abyme qui reflète la poétique de la plupart des romans sur la Grande Guerre, tout en tirant les conséquences des critiques formulées et en prenant même systématiquement, par leur pratique littéraire, le contrepied de toute une littérature héroïque et esthétisante qu'ils essaient de démystifier. Ainsi, de nombreux romans sur la Grande Guerre sont marqués par un conflit interne qui les incite à combattre toute tentative d'esthétisation de la guerre. S'ils décrivent un bombardement comme un feu d'artifice, c'est justement pour en exposer la beauté dans le dessein de l'isoler et de l'exclure de leur propre écriture. Ils décrivent le feu d'artifice par refus de devenir eux-mêmes un simple feu d'artifice littéraire. La littérature de la Grande Guerre refuse d'être «belle», elle ne veut plus faire partie des « Belles Lettres ». C'est pour cette raison qu'elle se trouve dans une relation agonale avec sa propre dimension esthétique. Plutôt qu'une esthétique de l'horreur, de l'abject ou de la laideur, un certain nombre de romans de 14-18 opte pour une écriture par-delà le beau et le laid, se décidant de fait pour une véritable « anesthétique littéraire » aux traits caractéristiques suivants :

1. La constellation des personnages. Le héros classique est remplacé par une multiplicité de personnages, l'individu par une masse anonyme de soldats. Dans les romans de Barbusse, de Remarque, de Cendrars ou de Dorgelès, le lecteur est confronté à une foule de protagonistes. Barbusse et Cendrars consacrent de nombreuses pages à en faire les portraits individuels et à décrire les dialogues qu'ils engagent entre eux. Mais leur nombre excessivement élevé empêche le lecteur de les percevoir comme individus. De cette manière, ces romans étouffent d'emblée toute tentative d'héroïsation et tout héroïsme.

2. La structure de l'action. Le récit d'actions singulières est réduit au profit d'une description détaillée de la vie quotidienne dans les tranchées. Cette réduction va de pair avec le remplacement du singulatif - mode temporel par excellence des actes héroïques - par l'itératif des actions répétées forgées par l'habitude et plus propices à raconter la vie quotidienne des soldats. De plus, la narration des actions humaines est en grande partie relayée par la description des canons et des mitrailleuses qui semblent tirer eux-mêmes sans l'aide d'aucun acteur humain. L'événement perd de l'importance, y compris celui qui semble pourtant crucial dans un récit de guerre, c'est-à-dire la mort du soldat. Ainsi, Barbusse, qui s'attarde pourtant sur ses personnages et leur vie de tous les jours, évoque leur mort d'une manière assez prosaïque. Cendrars, quant à lui, décrit longuement chacun des siens et souligne son importance en donnant son nom comme titre au chapitre qui lui est consacré. Mais les titres indiquent souvent le lieu où ils sont 
morts : Rossi (tué à Tillaloy ${ }^{54}$ ), Lang (tué à Bus ${ }^{55}$ ), Robert Bellesort (mort en Angleterre) et Segouana (tué à la ferme de Navarin ${ }^{56}$ ), Bikoff (aveugle de guerre, suicidé ${ }^{57}$ ), etc. Cendrars raconte également tous les détails de leur mort, surtout si ceux-ci sont peu glorieux :

\begin{abstract}
À Dompierre, ce sont les hommes qui volaient en l'air par sections entières, soufflés qu'ils étaient par les terrifiantes explosions des fourneaux de mines qui partaient en chapelet et beaucoup d'hommes ne retombaient pas, sinon sous forme de pluie de sang. ${ }^{58}[\ldots]$

Angéli était mort asphyxié, la tête dans du caca allemand, les jambes au ciel. ${ }^{59}$
\end{abstract}

3. Impassibilité. Ce qui frappe dans ces descriptions et dans bien d'autres, c'est leur caractère prosaïque et impassible, qui constitue un élément commun entre les récits de Barbusse, Dorgelès, Genevoix, Cendars et Remarque, comme si les auteurs voulaient empêcher le lecteur de s'identifier aux protagonistes ou bien de compatir à leurs sentiments. Chez Ernst Jünger, cette impassibilité dans la description de la mort atteint son comble ${ }^{60}$. Mais ce n'est là que la forme très marquée d'une attitude qui se retrouve également dans d'autres récits de 14-18 et qui sert surtout à empêcher le lecteur d'éprouver des émotions à l'égard des personnages, comme c'était encore le cas dans les romans de guerre héroïques.

4. Un degré anesthétique de l'écriture. En effet, le prosaïsme et le laconisme notoires des romans de la Grande Guerre peuvent se comprendre comme une écriture au sens que Roland Barthes a donné à ce concept dans son essai Le degré zéro de l'écriture, c'est-à-dire comme une prise de position ou un engagement qui se fait par et dans la forme littéraire même ${ }^{61}$. Barthes choisit le terme de « degré zéro » pour caractériser une écriture transparente qui ne se désigne pas comme littéraire ou artistique, mais qui «dépasse la Littérature en se confiant à une sorte de langue basique » ${ }^{62}$. Dans le cas concret des romans de guerre, cette écriture s'oppose à toute tentative d'embellissement et d'esthétisation de la guerre et se conçoit comme un souci

${ }^{34}$ Cendrars, La Main coupée, p. 23.

Ibid., p. 34.

${ }^{36}$ Ibid., p. 39.

Ibid., p. 105.

Ibid., p. 331

Ibid., p. 335.

${ }^{60}$ «Fortwährend zischte es in langem, scharfem Bogen heran, Blitze wirbelten den Boden der Lichtung hoch. "Sanitäter!" Wir hatten den ersten Toten. Dem Füsilier S. zerriß eine Schrapnelkugel die Halsschlagader. » (JÜNGER, In Stahlgewittern, p. 15).

Et, beaucoup plus loin : «Wir packten die aus den Trümmern ragenden Gliedmaßen und zogen die Leichen heraus. Dem einen war der Kopf abgeschlagen und der Hals saß am Rumpf wie ein großer, blutiger Schwamm. Aus dem Armstumpf des zweiten ragte der zersplitterte Knochen, und die Uniform war vom Blute einer großen Brustwunde durchtränkt. Dem dritten quollen die Eingeweide aus dem aufgerissenen Leib. » (Ibid., p. 101).

${ }^{61}$ Roland BARTHES, Le degré zéro de l'écriture suivi de Nouveaux essais critiques, Paris, Seuil, 1972

62 Ibid., p. 56. 
d'authenticité et de démystification. En fait, il s'agit là d'un parti pris contre l'écriture esthétique, un peu comme si le roman devait se racheter, parce qu'il décrit la guerre, par une écriture proche du style de ceux qui la font, c'est-àdire des journaux intimes et des lettres des poilus. Ce parti pris pour un « degré anesthétique » de l'écriture explique également pourquoi ces romans sont décevants quand on les compare, par exemple, aux œuvres d'avant-garde ou bien aux traitements modernes de la même thématique, tels L'Acacia de Claude Simon.

Que l'esthétisation de la guerre à laquelle s'oppose cette forme anesthétique soit loin d'être gratuite devient évident, quand on sait que Charles Péguy a trouvé la mort parce que, malgré les mises en garde répétées de ses camarades, il refusait de se coucher par terre, mais persistait à rester héroïquement debout face aux mitrailleuses allemandes ${ }^{63}$. Et si les romans de Dorgelès, Barbusse, Chevallier ou Remarque prennent systématiquement le contrepied de cette esthétique de la guerre en prônant une "anesthétique agonale ", c'est parce qu'ils veulent démystifier après coup cette esthétisation mensongère, c'est en somme parce qu'ils font la guerre contre la guerre par la littérature. Comme s’ils disaient à Péguy : «Profil bas ! Couche-toi par terre !»

${ }^{63}$ Romain Rolland, Charles Péguy, t. II, Paris, Albin Michel, 1944, p. 102. 